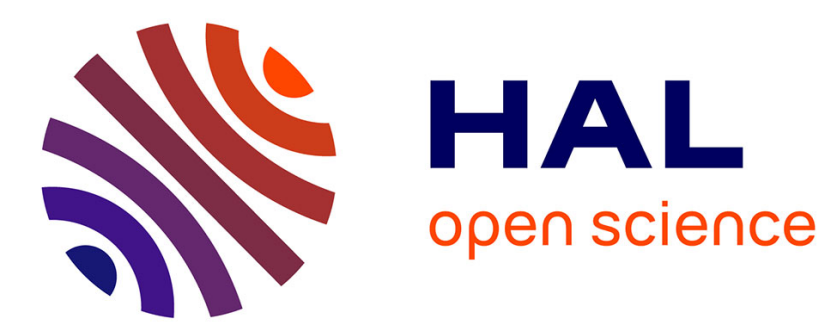

\title{
Etude comparée des gavages traditionnel et pneumatique chez l'oie et le canard
}

G. Guy, J.-C. Blum, N. Lapierre, David Gourichon

\section{To cite this version:}

G. Guy, J.-C. Blum, N. Lapierre, David Gourichon. Etude comparée des gavages traditionnel et pneumatique chez l'oie et le canard. Productions Animales, 1994, 7 (3), pp.169-175. hal-00896084

\section{HAL Id: hal-00896084 \\ https://hal.science/hal-00896084}

Submitted on 1 Jan 1994

HAL is a multi-disciplinary open access archive for the deposit and dissemination of scientific research documents, whether they are published or not. The documents may come from teaching and research institutions in France or abroad, or from public or private research centers.
L'archive ouverte pluridisciplinaire HAL, est destinée au dépôt et à la diffusion de documents scientifiques de niveau recherche, publiés ou non, émanant des établissements d'enseignement et de recherche français ou étrangers, des laboratoires publics ou privés. 
INRA Prod. Anim., 1994, 7 (3), $169-175$.
G. GUY, J.-C. BLUM*, N. LAPIERRE, D. GOURICHON

INRA Station expérimentale des Palmipèdes à Foie Gras Artiguères 40280 Benquet

*INRA Station de Recherches Avicoles 37380 Nouzilly

\section{Résumé}

Deux essais ont été mis en place à la Station expérimentale INRA des Palmipèdes à Foie Gras (Artiguères) afín de comparer les performances des oies et des canards soumis au gavage traditionnel au grain ou au gavage pneumatique utilisant du maïs broyé additionné d'eau. Cette dernière technique est très rapide mais semble donner des performances irrégulières et entraîne une moins bonne assimilation du maïs.

Au cours de l'essai 1, l'efficacité du "gavage pneumatique" basé sur l'emploi de farine injectée sous forme de bouillie dans l'oesophage à l'aide d'une pompe pneumatique est comparée à celle du gavage traditionnel (maïs grain) réalisé selon deux modalités : gavage intensif et gavage conduit plus lentement pour correspondre sensiblement au niveau d'ingestion alimentaire permis par le gavage pneumatique.

Ce schéma a été appliqué au canard mulard préparé au gavage (âge : 12 semaines) en constituant trois lots de 45 sujets chacun : lot CI (gavage traditionnel intensif), lot CD (gavage traditionnel "à la dose") et lot CPn (gavage pneumatique). Gavés pendant 13 jours, ces 3 lots consomment respectivement 10,$3 ; 9,4$ et $9,8 \mathrm{~kg}$ de maïs sec par canard, ce qui permet une augmentation de poids vif de 54 ; 50 et $44 \%$ avec production de foies gras pesant en moyenne : 698; 551 et $461 \mathrm{~g} / \mathrm{canard}$. Les différences de performances entre lots, toutes significatives $(P<0,01)$, placent systématiquement le lot $\mathrm{CPn}$ bon dernier : la consommation est plus élevée que dans le lot CD et le maïs utilisé moins efficacement.

Le schéma expérimental adopté pour l'oie prolonge le gavage pneumatique et par voie de conséquence le gavage traditionnel lent qui lui est associé. Les 3 lots comprennent chacun 30 oies âgées de 13 semaines. Les lot OI (gavage traditionnel intensif), OR (gavage traditionnel ralenti) et OPn (gavage pneumatique), gavés pendant 12 (OI) ou 18 jours (OR et $\mathrm{OPn}$ ), consomment respectivement 11,$3 ; 14,0$ et $16,7 \mathrm{~kg}$ de maïs sec par oie, ce qui permet une augmentation de poids vif de $44 ; 50$ et $55 \%$ avec un poids moyen de foie gras de $708 ; 747$ et 806 g/oie. La forte consommation imposée par gavage pneumatique a donc permis de bonnes performances, mais au prix d'une mortalité élevée (23\%) et d'une diminution du rendement alimentaire.

L'essai 2 analyse l'influence des deux types de gavage sur l'utilisation digestive. Chez le canard, l'énergie métabolisable du maïs ingéré ad libitum, par gavage traditionnel ou par gavage pneumatique, est trouvée identique quel que soit le niveau d'ingestion (100 ou $300 \%$ du niveau ad libitum) ; cependant la mesure de l'excrétion des matières sèches dans les fientes met en évidence une accélération du transit au cours des premières heures suivant un repas par gavage pneumatique. Chez l'oie, l'énergie métabolisable du maïs n'est pas trouvée différente après ingestion de maïs en grain (gavage traditionnel) ou de bouillie (gavage pneumatique) ; mais, quelle que soit la présentation, l'énergie métabolisable diminue significativement d'environ $5 \%$ lorsque le niveau d'ingestion passe de 100 à $300 \%$; la quantité totale de matières sèches excrétée dans les fientes est augmentée après un gavage pneumatique comparé au gavage traditionnel, mais cette augmentation apparaît tardivement après 24 heures et surtout 48 heures de jeûne. Les conséquences de ces observations sur les rendements alimentaires sont discutées.

\section{Etude comparée des gavages traditionnel et pneumatique chez l'oie et le canard}

Traditionnellement, le gavage est réalisé avec du maïs entier en grain, salé, cuit à l'eau (le maïs incorpore ainsi $30 \%$ d'eau) et additionné, au dernier moment, d'une très faible quantité de graisse pour faciliter le glissement des grains dans l'œesophage. Opération exigeant autrefois une longue durée (plus d'un mois chez l'oie), le gavage a été considérablement raccourci par les méthodes dites "intensives". Préparés au gavage pendant la période d'élevage, canards et oies sont gavés en moins de 2 semaines. Les performances en foie sont satisfaisantes et s'accompagnent d'un engraissement périphérique modéré. La faible consommation pendant le gavage indique un bon rendement alimentaire. (Babilé 1989 , Rousselot 1990, Guy et al 1994).

Cependant, les gavages traditionnels les plus intensifs ne font pas l'unanimité. Les animaux n'ayant pas tous la même capacité d'ingestion, le gaveur doit déterminer le meilleur programme pour chacun d'entre eux. C'est un travail délicat et astreignant où les erreurs sont coûteuses (mortalité). Les gavages plus lents sont souvent préférés, particulièrement chez l'oie. Chez le canard, on a préconisé un gavage programmé susceptible de convenir à tous les canards, c'est le gavage "à la dose" qui atténue les différences individuelles, en tous cas celles qui sont liées à l'ingestion alimentaire ; les manipulations étant invariables d'un canard à l'autre, l'exécution du travail est aussi plus rapide (Robin et Castaing 1993).

Le gavage pneumatique, réalisé avec une pompe pneumatique qui injecte le maïs broyé et humidifié sous forme d'une bouillie épaisse dans l'oesophage, est apparu plus récemment. Pour que la pâte soit suffisamment fluide, il faut un apport d'eau relativement élevé $(50 \%)$, ce qui interdit les gavages très intensifs. C'est le système de gavage réputé le plus rapide de tous. A notre connaissance, aucune étude précise ne lui a été consacrée sur le plan technique. 
Nous avons voulu comparer l'efficacité des gavages pneumatique et traditionnel (essai 1), ce dernier étant réalisé selon deux modalités : gavage intensif (le plus rapide possible) et gavage plus lent suivant un rythme d'alimentation que nous avons voulu proche de celui autorisé par le gavage pneumatique afin de disposer d'une base de comparaison. Par ailleurs, une étude préliminaire du gavage pneumatique indiquant un rendement alimentaire diminué, nous avons réalisé parallèlement (animaux provenant des mêmes troupeaux) un autre essai (essai 2) pour analyser l'influence du gavage sur l'utilisation digestive du maïs.

\section{1 / Conditions expérimentales}

Le même mais (variété Fuji blanc provenant d'un seul lot) a été utilisé dans tous les essais qui font l'objet de cette étude.

Pour le gavage traditionnel, ce maïs, conservé en graines entières, additionné de $1 \%$ de

Tableau 1. Conditions expérimentales de l'essai 1. Canards mulards.

\begin{tabular}{|lccc|}
\hline Lot & CI & CD & CPn \\
\hline Effectif & 45 & 45 & 45 \\
Maïs & grain & grain & broyé \\
Durée (j) & 13 & 13 & 13 \\
Nb repas/j & 2 & 2 & 3 \\
Gavage & intensif & modéré & modéré \\
Quantité & liée à & & \\
de maïs & l'animal & prétablie & préétablie \\
\hline
\end{tabular}

Tableau 2. Conditions expérimentales de l'essai 1. Oies.

\begin{tabular}{|c|c|c|c|}
\hline Lot & OI & OR & OPn \\
\hline Effectif & 30 & 30 & 30 \\
\hline Maïs & grain & grain & broyé \\
\hline Durée (j) & 12 & 18 & 18 \\
\hline $\mathrm{Nb}$ repas/j & 5 & 4 & 6 \\
\hline Gavage & intensif & lent & modéré \\
\hline $\begin{array}{l}\text { Quantité } \\
\text { de mais }\end{array}$ & $\begin{array}{l}\text { liée à } \\
\text { l'animal }\end{array}$ & $\begin{array}{l}\text { liée à } \\
\text { l'animal }\end{array}$ & préétablie \\
\hline
\end{tabular}

Tableau 3. Conditions expérimentales de l'essai 2.

\begin{tabular}{|lcl|}
\hline Lot & Effectif & Traitement \\
\hline Témoin & 7 & Mais granulé, alimentation ad libitum, ingestion 100\% \\
GT 100 & 7 & Mais grain, gavage traditionnel, ingestion 100\% \\
GT 300 & 7 & Mais grain, gavage traditionnel, ingestion 300\% \\
GPn 100 & 7 & Maïs granulé + eau, gavage pneumatique, ingestion 100\% \\
GPn 300 & 7 & Mais granulé + eau, gavage pneumatique, ingestion $300 \%$ \\
\hline
\end{tabular}

chlorure de sodium, est cuit à l'eau jusqu'à ébullition en suivant la technique classique sauf que, pour cette étude, on n'ajoute aucune graisse de façon à disposer de régimes de gavage tous comparables par leur composition.

Pour le gavage pneumatique, le maiis est d'abord broyé. La farine est additionnée de $0,5 \%$ de chlorure de sodium, puis granulée (5 mm) à la vapeur (Landal Maïsadour, 40090 Haut Mauco). Les granulés sont transformés au moment de l'emploi en une pâte fluide par addition d'eau : $50 \%$ de granulés et $50 \%$ d'eau à $40^{\circ} \mathrm{C}$.

\section{1 / Essai 1}

Les 135 canards mulards mâles (Sica Sepalm), préparés au gavage selon la technique précédemment décrite, sont placés en cages individuelles de gavage à l'âge de 12 semaines. Ils sont répartis en 3 lots gavés pendant 13 jours (tableau 1).

Les 90 oisons mâles (Sica Sepalm), préparés au gavage (Guy et al 1994) sont placés à l'âge de 13 semaines en cages individuelles de gavage et réparties en 3 lots (tableau 2).

Tous les animaux sont pesés avant le gavage et à la fin de celui-ci.

Les carcasses sont éviscérées "à chaud", c'est à dire immédiatement après l'abattage et la plumaison (Rousselot-Pailley 1990). Le foie est pesé, puis conservé à $+4^{\circ} \mathrm{C}$ pendant 24 heures. On prélève alors un échantillon de $60 \mathrm{~g}$ de foie pour mesurer la fonte lipidique selon la méthode mise au point par RousselotPailley et al (1993) : poids de graisse exsudée $(\%)$ au cours du chauffage à $105^{\circ} \mathrm{C}$ pendant 50 minutes.

\section{2 / Essai 2}

L' essai 2 est réalisé parallèlement à l'essai 1 en faisant appel aux mêmes bandes d'élevage d'oies et canards préparés au gavage. Les animaux sont retirés des troupeaux au moment où devrait commencer le gavage, puis placés dans des cages permettant des mesures de bilan (contrôle de l'ingestion et de l'excrétion), afin de déterminer l'énergie métabolisable du mais en fonction du niveau d'ingestion et du mode de gavage. La méthode adoptée est voisine de celle proposée pour le coq par Kussaibati et Leclercq (1985). Les animaux sont entraînés à la consommation du maïs pendant 48 heures, selon des conditions identiques (forme de présentation, quantité de maïs) à celles qu'ils subissent lors de l'expérimentation. Cette période d'adaptation est suivie par un jeûne de 18 heures. La mesure de l'énergie métabolisable proprement dite s'étend sur 48 heures : les animaux sont alimentés pendant 30 heures, puis de nouveau placés au jeûne pendant 18 heures ; on détermine la quantité d'aliment consommé pendant les 30 heures et les fientes excrétées au cours des 48 heures (alimentation suivie du jeûne final). L'alimentation est soit ad libitum (lot témoin sur maïs granulé), soit effectuée par gavage : 4 gavages au cours des 
30 heures chez le canard, 5 gavages chez l'oie, les quantités distribuées étant alors calculées pour que l'apport de maïs soit ou bien équivalent à celui ingéré en alimentation ad libitum, ou bien 3 fois supérieur. Au total, on dispose, chez l'oie comme chez le canard, de 5 lots de 7 animaux chacun, qui diffèrent par le mode d'alimentation et par le niveau d'ingestion (tableau 3 ).

L'énergie brute du maïs et des fientes lyophilisées est mesurée dans une bombe calorimétrique adiabatique. On calcule l'énergie métabolisable (EM) apparente non corrigée pour les différents lots en appliquant la formule classique :

$\mathrm{EM}(\mathrm{kcal} / \mathrm{kg}$ de matière sèche $)=(\mathrm{EB}$ ingérée - EB excrétée)/ Quantité de maïs sec ingéré

D'autre part, huit canards et huit oies préparés au gavage comme précédemment sont placés dans les cages individuelles permettant des mesures de bilan. Après 16 heures de jeûne, les animaux sont gavés avec un régime de gavage : 4 oies et 4 canards par gavage traditionnel avec du maïs grain, les autres par gavage pneumatique avec granulé + eau. Les quantités ingérées par chaque animal sont déterminées avec précision ; voisines, en équivalent maïs, de $170 \mathrm{~g}$, elles varient peu d'un individu à l'autre. Les fientes sont récoltées périodiquement après le repas (5 récoltes au cours de 12 premières heures, 1 récolte après 24 heures et une dernière récolte après 48 heures) et leur teneur en matière sèche est estimée.

\section{2 / Résultats}

\section{1 / Méthode de gavage et production du foie gras}

\section{a / Canards}

Les performances de production et la qualité du foie gras sont présentées au tableau 4. La mortalité est faible ou nulle dans les lots soumis au gavage peu intense (lots CD et $\mathrm{CPn}$ ), un peu plus élevée $(6,7 \%)$ pour le gavage traditionnel intensif. Identique à l'entrée en gavage pour tous les lots, le poids vif moyen augmente inégalement pour atteindre une valeur maximum dans le lot CI $(+54 \%)$, suivi par le lot CD $(+50 \%)$ et le lot CPn $(+44 \%)$, les différences entre lots étant toutes significatives. Cette variation du poids vif peut être attribuée, mais en partie seulement, aux différences de consommation : c'est le lot $\mathrm{CI}$ qui a consommé la plus forte quantité de maîs $(10,3 \mathrm{~kg})$, mais le lot CD $(9,4 \mathrm{~kg})$ est largement dépassé par le lot CPn $(9,8 \mathrm{~kg})$.

Le poids des foies gras place les lots suivant la même hiérarchie que les poids vifs au moment de l'abattage : $698 \mathrm{~g}$ dans le lot CI contre respectivement $551 \mathrm{~g}$ et $461 \mathrm{~g}$ dans les lots CD et CPn, toutes les différences étant significatives $(P<0,01)$. La dégradation du rendement alimentaire, particulièrement sensible dans le lot CPn, ressort de la comparaison des indices : indice de consommation et indice de production du foie gras (il faut $2,14 \mathrm{~kg}$ de maïs pour produire $100 \mathrm{~g}$ de foie gras dans le lot CPn contre $1,48 \mathrm{~kg}$ dans le lot CI). Quant à la fonte lipidique, elle augmente avec le poids du foie (Babilé 1989, Rousselot et al 1990), et est donc plus élevée dans le lot CI.

\section{b / Oies (tableau 5)}

La mortalité s'élève à $23,3 \%$ dans le lot OPn ( 2 morts +5 abattus d'urgence dont 3 seulement ont pu être récupérés) alors qu'elle est nulle dans les autres lots. Identiques dans les 3 lots à l'entrée en gavage, les poids vifs augmentent davantage dans les lots OPn $(+55 \%)$ et $\mathrm{OR}(+50 \%)$ où les gavages sont prolongés 18 jours que dans le lot OI $(+44 \%)$ qui est gavé seulement 12 jours, la différence entre OR et OI n'étant cependant pas significative. Les variations de poids vif sont en relation avec la quantité de maïs consommée, quantité plus élevée dans le lot OPn $(16,7 \mathrm{~kg} / \mathrm{oie})$ que dans les lots OR (14 kg) et OI (11,3 kg).

La variabilité des poids de foie $(\mathrm{CV}=$ $25,5 \%$ ) explique qu'aucune différence entre lots ne soit significative, bien que les foies soient nettement plus lourds dans le lot OPn (806 g) que dans le lot OI (708 g). Chez l'oie comme chez le canard, c'est le gavage intensif (lot OI) qui assure les meilleurs rendements alimentaires et le gavage pneumatique (lot $\mathrm{OPn}$ ) les plus mauvais : 4,6 vs 5,4 pour l'indice de consommation; 1,60 vs 2,07 pour l'indice de production du foie gras ( $\mathrm{kg}$ de maïs/100 $\mathrm{g}$ de foie).

\section{2 / Influence du mode de gavage sur la valeur énergétique du maïs}

Les valeurs d'énergie métabolisable du maïs mesurées chez l'oie et chez le canard dans les différents lots sont rapportés au tableau 6 .

Chez le canard, le mode de gavage et le niveau d'ingestion influencent peu les résultats obtenus, aucune différence n'étant significative. On constate tout au plus une légère tendance : qu'ils soient ingérés à la pompe (+ eau) ou ad libitum, les granulés de maïs apporteraient un peu plus d'énergie métabolisable que le maïs en grains entiers. La difficulté des mesures de consommation (régurgitation après gavage et surtout gaspillage en alimentation ad libitum) explique la variabilité des mesures, reflétée dans la valeur élevée des écarts-types, particulièrement dans le lot témoin. C'est une difficulté rencontrée par tous les expérimentateurs, qui rend imprécise la détermination de l'énergie métabolisable chez le canard (Mohamed et al 1989).

Chez l'oie, le niveau d'ingestion a plus d'effet que le mode de gavage sur la valeur EM qui est significativement réduite pour les apports alimentaires importants de $300 \%$ comparés à $100 \%$. Pour les mêmes raisons que chez le canard, la variabilité des mesures est élevée, particulièrement dans le lot GPn300, l'oie supportant mal une ingestion élevée de 
Tableau 4. Influence du mode de gavage des canards sur les performances de production et la qualité du foie gras (essai 1). Moyennes \pm écarts-type.

\begin{tabular}{|lccc|}
\hline Lot & CI & CD & CPn \\
\hline Effectif initial & 45 & 45 & 45 \\
Durée du gavage (j) & 13 & 13 & 13 \\
Nombre de repas & 25 & 25 & 36 \\
Nombre de morts & 3 & 1 & 0 \\
Poids vif avant gavage (g) & $4289 \pm 332^{\text {a }}$ & $4194 \pm 292^{\text {a }}$ & $4239 \pm 288^{\text {a }}$ \\
Poids vif à l'abattage (g) & $6621 \pm 492^{\mathrm{a}}$ & $6309 \pm 392^{\mathrm{b}}$ & $6118 \pm 269^{\mathrm{c}}$ \\
Poids du foie (g) & $698 \pm 101^{\mathrm{a}}$ & $551 \pm 93^{\mathrm{b}}$ & $461 \pm 117^{\mathrm{c}}$ \\
Fonte lipidique (\%)(1) & $45,1 \pm 10,1^{\mathrm{a}}$ & $35,3 \pm 10,3^{\mathrm{h}}$ & $30,8 \pm 13,1^{\mathrm{b}}$ \\
Consommation (g) & $(2)$ & 9389 & 9841 \\
Indice de consommation $^{(3)}$ & 10313 & 4,4 & 5,2 \\
Indice de prod. foie $^{(4)}$ & 4,4 & 1,70 & 2,14 \\
\hline
\end{tabular}

Les moyennes suivies de lettres différentes sont significativement différentes.

(1) Fonte lipidique du foie gras en \% du poids de foie.

(2) Consommation en g de maïs sec (grains ou granulés) pendant le gavage.

(3) Indice de consommation = consommation/gain de poids pendant le gavage

(4) Indice de production du foie gras = maïs sec ingéré en $\mathrm{kg}$ pour $100 \mathrm{~g}$ de foie gras

Tableau 5. Influence du mode de gavage des oies sur les performances de production et la qualité du foie gras (essai 1). Moyennes \pm écarts-type.

\begin{tabular}{|c|c|c|c|}
\hline Lot & OI & OR & OPn \\
\hline Effectif initial & 30 & 30 & 30 \\
\hline Durée du gavage (j) & 12 & 18 & 18 \\
\hline Nombre de repas & 54 & 70 & 102 \\
\hline Nombre de morts & 0 & 0 & 7 \\
\hline Poids vif avant gavage (g) & $5511 \pm 406^{\mathrm{a}}$ & $5522 \pm 402^{\prime}$ & $5538 \pm 409^{a}$ \\
\hline Poids vif à l'abattage (g) & $7946 \pm 549^{b}$ & $8267 \pm 667^{\text {ib }}$ & $8594 \pm 613^{a}$ \\
\hline Poids du foie (g) & $708 \pm 224^{2}$ & $747 \pm 167^{a}$ & $806 \pm 177^{a}$ \\
\hline Fonte lipidique $(\%)^{\prime 1 /}$ & $18,6 \pm 12,1^{\text {* }}$ & $18,0 \pm 7,9^{a}$ & $22,0 \pm 10,1^{\mathrm{a}}$ \\
\hline Consommation $(\mathrm{g})^{|2|}$ & 11300 & 14048 & 16704 \\
\hline Indice de consommation ${ }^{(3)}$ & 4,6 & 5,1 & 5,4 \\
\hline Indice de prod. foie $\mathrm{e}^{(4)}$ & 1,60 & 1,88 & 2,07 \\
\hline
\end{tabular}

(1), (2), (3) et (4) : voir tableau 4.

Tableau 6. Energie métabolisable du maïs (kcal/kg de matière sèche, moyenne \pm écart-type) mesurée chez le canard et l'oie en faisant varier la présentation (maïs en grains, bouillie ou granulés secs) et le niveau d'ingestion (100 ou $300 \%$ de la consommation ad libitum).

\begin{tabular}{|lccccc|}
\hline $\begin{array}{l}\text { Présentation du maïs } \\
\text { Niveau d'ingestion }\end{array}$ & $\begin{array}{c}\text { grains } \\
\mathbf{3 0 0} \%\end{array}$ & $\begin{array}{c}\text { grains } \\
\mathbf{1 0 0} \%\end{array}$ & $\begin{array}{c}\text { bouillie } \\
\mathbf{3 0 0} \%\end{array}$ & $\begin{array}{c}\text { bouillie } \\
\mathbf{1 0 0} \%\end{array}$ & $\begin{array}{c}\text { granulés } \\
\text { ad libitum }\end{array}$ \\
\hline Canards & $3712^{\mathrm{a}}$ & $3669^{\mathrm{a}}$ & $3757^{\mathrm{a}}$ & $3753^{\mathrm{a}}$ & $3768^{\mathrm{a}}$ \\
& \pm 46 & \pm 94 & \pm 41 & \pm 52 & +181 \\
Oies & $3560^{\mathrm{b}}$ & $3673^{\mathrm{a}}$ & $3541^{\mathrm{b}}$ & $3752^{\mathrm{a}}$ & $3750^{\mathrm{a}}$ \\
& \pm 47 & \pm 74 & \pm 160 & \pm 39 & +73 \\
\hline
\end{tabular}

Pour chaque type de palmipède, les valeurs qui ne sont pas suivies de la mème lettre diffèrent significativement entre elles $(\mathrm{P}<0,05)$. 
bouillie. Comme chez le canard alimenté à $100 \%$, la valeur énergétique est plus élevée quand le maïs est granulé (lots témoin et GPn100 vs GT100).

\section{3 / Cinétique de l'excrétion de la matière sèche des fientes après un gavage}

L'évolution des quantités excrétées (quantités cumulées par $100 \mathrm{~g}$ de maïs ingéré sec) chez le canard gavé traditionnellement ou avec la pompe pneumatique est présentée sur la figure 1. Les cinétiques sont voisines pour les deux modes de gavage; toutefois l'excrétion de la matière sèche est significativement plus rapide au cours des premières heures suivant le gavage à la pompe ; la différence n'est plus significative après 24 heures et inexistante après 48 heures.

Chez l'oie, l'excrétion est plus lente que chez le canard au cours des premières heures et il n'y a pas de différence entre les deux modes de gavage. Mais, après 24 heures, la quantité totale excrétée est significativement plus élevée chez les oies qui ont consommé la bouillie à la pompe; la différence s'accuse après 48 heures.

\section{3 / Discussion}

\section{1 / Canards}

Chez le canard, le gavage traditionnel intensif en 12 jours a permis des performances élevées avec des poids de foie très importants ; il faut cependant prendre en compte la mortalité en relation avec la délicate appréciation, laissée au gaveur, de la quantité de maïs à distribuer pour tirer le meilleur parti de chaque animal. Le gavage "à la dose" est moins exigeant : programmé à l'avance, il peut être exécuté rapidement avec des risques limités. Les performances obtenues sont moins bonnes; les foies sont moins gros (147 $\mathrm{g}$ de foie en moins par canard) et, de ce fait, fondent moins (avantage qui compense incomplètement la perte quantitative). Le gavage pneumatique fournit les foies les plus petits avec des rendements alimentaires de production nettement diminués. Comparé au lot "à la dose", le lot CPn produit $90 \mathrm{~g}$ de foie en moins par canard, tout en consommant plus. Force est d'admettre une mauvaise utilisation du maìs distribué après broyage et granulation.

Pourtant, l'étude de la valeur énergétique du mais n'a montré aucune différence significative liée au mode de gavage ou au niveau d'ingestion. Chez le coq, l'énergie métabolisable est souvent augmentée par la granulation à la vapeur par suite de la gélatinisation du grain d'amidon (Mercier et Guilbot 1974), ce qui expliquerait que la farine soit plutôt mieux utilisée (tendance non significative) que le maïs en grains. La cinétique d'excrétion des matières sèches mesurée dans les fientes après un repas important ( $170 \mathrm{~g}$ de maïs) paraît accélérée par l'ingestion de bouillie comparée
Figure 1. Evolution de la quantité cumulée de matière sèche (MS) excrétée après un repas.

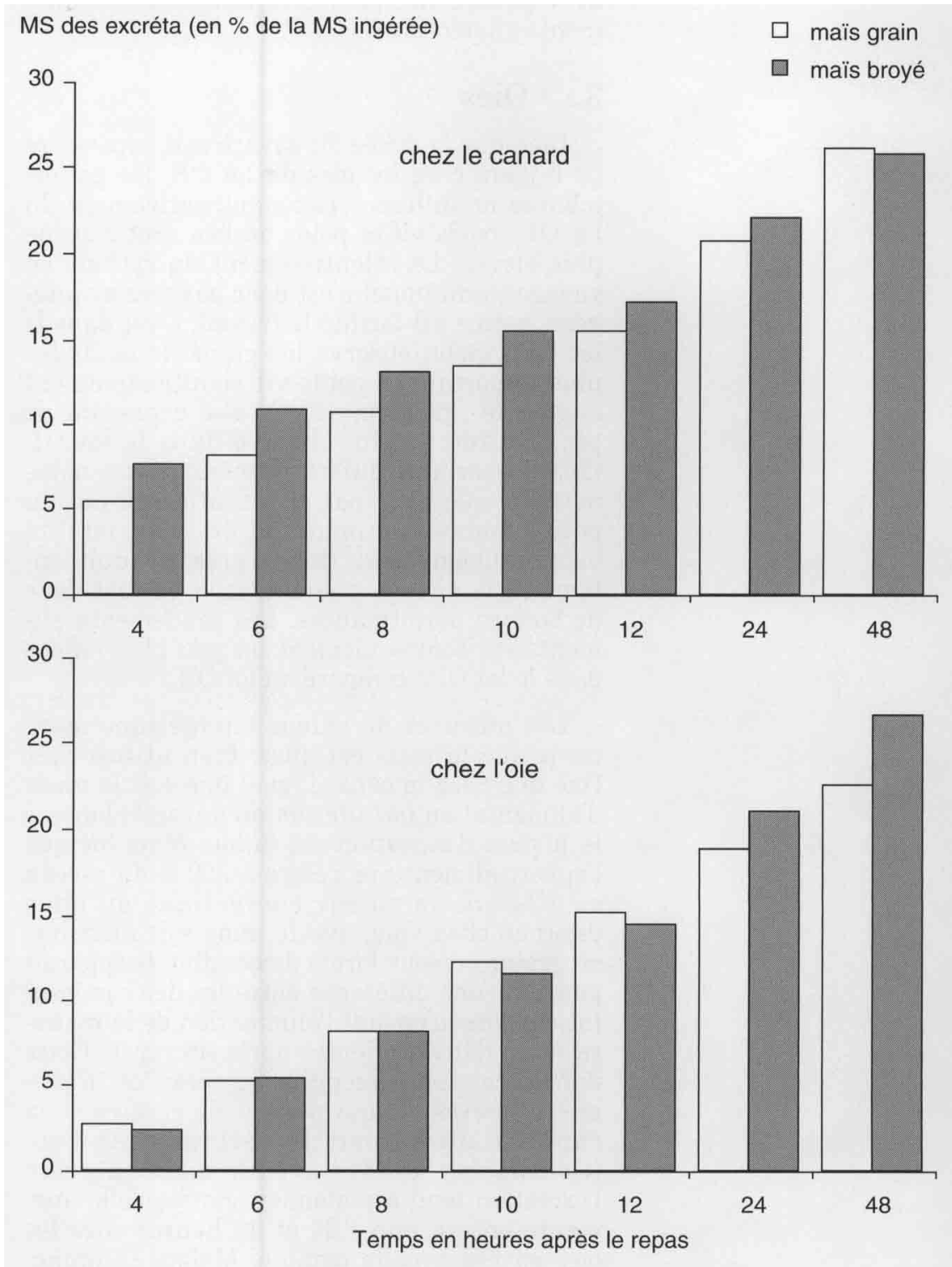

au maïs en grains. Cette accélération du transit a déjà été observée chez le canard commun disposant d'un aliment broyé complet ingéré à $250 \%$ du niveau ad libitum sous forme de pâte après addition d'eau (Zhou et al 1989). Dans ce cas, la digestibilité de la matière organique mesurée en séparant fèces et urine (anus artificiel) était diminuée de $3,6 \%$. La mesure faite dans cette étude est plus globale et moins précise (non corrigée pour l'azote excrété dans l'urine) ; elle concerne aussi un aliment différent, réputé hautement digestible, qui peut être moins affecté par l'accélération du transit qu'un régime complexe. Cependant, la diminution des rendements alimentaires chez les canards gavés à la pompe, laisse supposer qu'une différence doit finir par s'établir chez l'animal suralimenté par les gavages successifs. La digestion des lipides serait améliorée, mais celle de l'amidon, source énergétique majeure du maïs, serait réduite (Leclercq et al 1973). Il faudra de nouveaux essais pour savoir 
dans quelles conditions et à quel moment au cours du gavage, la digestibilité se dégrade avec pour conséquence la baisse des rendements alimentaires.

\section{2 / Oies}

Bien que la durée du gavage soit supérieure de 6 jours chez les oies du lot OR, les performances ne different pas significativement du lot OI : poids vif et poids de foie sont à peine plus élevés. Le ralentissement du rythme en gavage traditionnel n'est donc pas très avantageux, même s'il facilite le travail. C'est dans le lot OPn qu'on observe les gains de poids les plus importants : poids vif significativement augmenté ; poids moyen du foie dépassant de plus de $100 \mathrm{~g}$ celui observé dans le lot OI. Cette importante différence mérite d'être notée même si elle n'est pas significative du fait du petit nombre d'animaux et de la variabilité bien connue du poids du foie gras. Conduit lentement, le gavage pneumatique permet donc de bonnes performances. Les rendements alimentaires sont seulement un peu plus faibles dans le lot OPn comparé au lot OR.

Les mesures de valeur énergétique montrent que le maïs est aussi bien utilisé chez l'oie que chez le canard, quel que soit le mode d'alimentation (ad libitum ou gavage), lorsque le niveau d'ingestion est faible. Mais lorsque l'apport alimentaire s'élève à $300 \%$ du niveau ad libitum, la valeur énergétique du maïs diminue chez l'oie, que le maiis soit distribué en grains ou sous forme de bouillie. Il apparaît pourtant une différence entre les deux présentations lorsqu'on suit l'élimination de la matière sèche dans les fientes après un repas. Cette différence n'est perceptible qu'après les 16 première heures, ce qui permet de confirmer la rapidité du transit intestinal chez l'oie (Courboulay 1988) : avec le maïs en grain l'excrétion tend à plafonner, alors qu'elle augmente encore après 24 et 48 heures chez les oies gavées avec la bouillie. Malgré l'élimination prolongée après un repas de gavage volumineux sous forme de bouillie, la mesure de l'énergie métabolisable chez les oies du lot GPn 300 peut être considérée comme valide. En effet, durant la phase préparatoire, les niveaux d'alimentation ont été identiques à ceux de la phase expérimentale. Toutefois, cette élimination prolongée pourrait correspondre à la rétention d'une partie du repas dans la partie antérieure du tube digestif expliquant les difficultés rencontrées (mortalité élevée) lors du gavage des oies avec la pompe.

\section{Conclusion}

Le principal avantage apporté par le gavage pneumatique est un gain sur le temps de travail. D'après Everlet (1990), le temps total de travail pendant la période de gavage, englobant les préparations et nettoyages ainsi que les soins aux animaux serait de 13,3 minutes par canard avec le système pneumatique, contre 21,8 minutes avec le système traditionnel ; l'avantage serait moins évident pour l'oie avec un temps de 1 heure contre 1 heure 17 . Ces chiffres n'ont bien sûr qu'une valeur indicative; ils dépendent de l'habileté des gaveurs pris comme référence et sont susceptibles d'évoluer considérablement avec la méthodologie, du gavage pneumatique comme du gavage traditionnel.

Nos résultats montrent que le gavage pneumatique, comparé au gavage traditionnel, oblige à une conduite moins intensive, ce qui se traduit par un foie plus petit, ou un gavage plus long, ou bien encore un compromis entre ces deux options. Le foie plus petit fondant moins, on gagne en qualité, ce qui peut être appréciable dans le cas du foie de canard particulièrement fondant.

Les rendements alimentaires paraissent plus faibles pour les gavages pneumatiques. Mais, là encore, des améliorations peuvent être apportées. Il faudra préciser la finesse optimum de la mouture et le mode de préparation de la bouillie, il faudra aussi déterminer la conduite et la durée optimum du gavage.

En ce qui concerne les mesures d'énergie métabolisable, il faut demeurer prudent sur son interprétation. En effet, nous ne constatons pas de différences liées à la forme de présentation du maïs. Ces mesures ayant été ponctuelles ne sont peut être pas représentatives de l'ensemble du gavage. C'est une hypothèse qu'il conviendra de vérifier ultérieurement. 


\section{Références bibliographiques}

Babilé R., 1989. La production de foies gras de canards de Barbarie (Cairina Moschata) : aspects génétiques, nutritionnels, technologiques. Thèse de Doctorat d'Etat, Institut National Polytechnique de Toulouse.

Courboulay V., 1988. Validation de la méthode de gavage humide chez le coq et l'oie adultes. Mémoire fin d'études, $47 \mathrm{pp}$, ENSA Rennes et INRA Rech. Avicoles, Nouzilly.

Everlet P., 1990. La gaveuse pneumatique : résultats objectifs du réseau gersois. ITAVI, Dax (40), 15 novembre.

Guy G., Rousselot-Pailley D., Gourichon D., 1994. Comparaison des performances quantitatives et qualitatives du gavage de l'oie, du canard mulard et du canard de Barbarie. Ann. Zootech. (en cours de publication)

Kussaibati R., Leclercq B., 1985. A simplified rapid method for the determination of apparent and true metabolisable energy values of poultry food. Arch. Geflügelk., 49, 54-62.

Leclercq B., Hassan I., Thivend P., Blum J.C., 1973. Utilisation comparée du maïs entier et du maïs broyé pour le gavage du canard. Ann. Zootech,.22, 337-340.
Mercier Ch., Guilbot, 1974. Influence des conditions de granulation du maïs sur les caractéristiques physicochimiques de son amidon. Ann. Zootech., 23, 241-251.

Mohamed K., Leclercq B. , Anwar A., El-Alaily H., Soliman H., 1984. A comparative study of metabolisable energy in duckling and domestic chicks. Anim. Feed Sci. Technol., 11, 199-210.

Robin N., Castaing J., 1993. Incidence du mais distribué en gavage sur les performances des canards mulards. 1ères Journées de la Recherche sur les Palmipèdes à Foie Gras, Bordeaux, 28 Avril.

Rousselot-Pailley D., 1990. Incidence des conditions d'abattage et de réfrigération sur la qualité des produits. Session ITAVI Dax (40), 15 novembre.

Rousselot-Pailley D., Sellier N., Gourichon D., 1990. Production comparée d'oies gavées deux ou trois fois par jour avec repasse. L'aviculteur, Juin, 50.

Rousselot-Pailley D., Rouvier R., Bartel G., 1993. Méthodologie du test de fonte des foies gras. lères Journées de la Recherche sur les Palmipèdes à Foie Gras, Bordeaux, 28 Avril.

Zhou Z-X., Issihiki Y., Yamauchi K-En, Nakahiro Y., 1989. Effects of feeding method and feed intake on the feed digestibility and activities of digestive enzymes in the feces of ducks. Jpn. Poult. Sci., 26, 354-361.

\section{Summary}

A comparative study of traditional and pneumatic force-feeding of geese and ducks.

Two trials were carried out at the Experimental Station of Artiguères to compare the performances geese and ducks when force-fed in a traditional program with corn grain or a new pneumatic system using wet ground corn. This new technique is very fast but seems to give irregular performance because assimilation of corn is reduced. During the first trial, efficiency of pneumatic cramming was compared with traditional force-feeding. Three experimental groups of forty five twelve week old mule ducks were fed as follows : intensive group (CI, traditional force-feeding); moderate group (CD, traditional force-feeding) ; pneumatic group (CPn, using ground corn).

The duration of the experiment was thirteen days. Maize consumption was respectively $10.3 \cdot 9.4$ and $9.8 \mathrm{~kg}$ of dry corn per duck on average. At the same time live body weight increase was $54 \cdot 50$ and $44 \%$ including "foies gras" of 698 - 551 and 461 grams. The differences recorded were all significant $(P<0.01)$, showing that the CPn group was the least productive in spite of a corn consumption higher than CD. Hence corn efficiency is affected in this technique.

For the geese trial we used three groups of thirty birds, thirteen weeks old : intensive group (OI, traditional, duration 12 days); moderate group (OR, traditional, duration 18 days) ; pneumatic group (OPn, with ground corn, duration 18 days).

Corn consumptions were respectively $11.3 \cdot \mathbf{1 4 . 0}$ and $16.7 \mathrm{~kg}$. They showed a body weight increase of $44-50$ and $55 \%$ including "foies gras" of 708 . 747 and 806 grams. "Foie gras" production of the OPn group was assured by the high level of corn consumed, but mortality was high $(23 \%)$ and food efficiency was affected.

During the second trial we compared corn digestive-utilisation according to its presentation. In both cases mule ducks showed no difference for the apparent metabolisable energy, and the level of ingestion at $100 \%$ or $300 \%$ of ad libitum was also without effect. However, measurement of feces excretion showed a transit acceleration during the first hours after the meal. Apparent metabolisable energy was not different whatever the presentation of corn for geese. In contrast, a high level of ingestion at $300 \%$ decreased significantly $5 \%$ apparent metabolisable energy in both presentations. Total feces excretion increased with the pneumatic technique but later after the meal at 24 hours and especially 48 hours.

GUY G., BLUM J-C., LAPIERRE N., GOURICHON D., 1994. Etude comparée des gavages traditionnel et pneumatique chez l'oie et le canard. INRA Prod. Anim., 7 (3), 169-175. 